\title{
Dewaxed Brown Rice Feed Improves Fatty Liver in Obese and Diabetic Model Mice
}

\author{
HIROYUKI INAGAWA ${ }^{1,2,3}$, TOSHIYUKI SAIKA ${ }^{4}$, NAOKI NISHIYAMA ${ }^{4}$, TAKASHI NISIZAWA ${ }^{5}$, \\ CHIE KOHCHI ${ }^{1,2,5}$, MAYA UENOBE $^{6}$ and GEN-ICHIRO SOMA ${ }^{1,2,3}$ \\ ${ }^{1}$ Control of Innate Immunity, Technology Research Association, Kagawa, Japan; \\ ${ }^{2}$ Faculty of Medicine, Kagawa University, Kagawa, Japan; \\ ${ }^{3}$ Research Institute for Healthy Living, Niigata University of Pharmacy and Applied Life Sciences, Niigata, Japan; \\ ${ }^{4}$ Toyo Rice Corp., Wakayama, Japan; \\ ${ }^{5}$ Macrophi Inc., Kagawa, Japan; \\ ${ }^{6}$ Faculty of Health and Human Development, University of Nagano, Nagano, Japan
}

\begin{abstract}
Background/Aim: Dewaxed brown rice has macrophage activation ability via TLR4 and contains a high amount of lipopolysaccharides (LPS). It is expected that dewaxed brown rice can help prevent lifestyle diseases. In this study, the anti-obesity effect of dewaxed brown rice was investigated using obese and diabetic model mice. Materials and Methods: Dewaxed brown rice and white rice were polished and powdered by Toyo Rice Co. Diet pellets were prepared (AIN-93) with 50\% dewaxed brown rice or white rice powder and fed to type II diabetic model KK-Ay mice for 10 weeks. Weight and fasting blood glucose were measured every week, and whole blood and liver was collected on the final day for the evaluation of biochemical data. Results: A $20 \%$ reduction in body weight was found in the dewaxed brown rice feed and white rice feed groups compared to the normal feed group. Fasting blood glucose increased in the normal-diet group, but on the other hand, the blood glucose in the white rice and the dewaxed brown rice feed group was almost constant. Dewaxed brown rice feed group of plasma ALT and AST, liver TG and T-CHO were significantly lower than that of the control and the white rice feed group. Conclusion: Dewaxed brown rice feed has an anti-obesity effect to suppress increasing body weight, fasting blood glucose, and an effect of suppressing fatty liver.
\end{abstract}

Brown rice has been reported as having anti-obesity properties (1). However, brown rice is known to convert

Correspondence to: Dr. Hiroyuki Inagawa, Control of Innate Immunity, Technology Research Association, 2217-16 FROMKAGAWA Bio Lab., Hayashi-cho, Takamatsu, Kagawa, 761-0301, Japan. Tel/Fax: +81 878139203, e-mail: pina@shizenmenki.org

Key Words: Brown rice, lipopolysaccharides, fatty liver. complete starch in cooking, thus it is not a popular food. The main reason for this is their wax bran layer existing in the outermost layer which excludes water absorption. Recently, rice milling technology has been established to remove only the wax bran layer (2). Because of peeling wax layer, this brown rice easily penetrates water into the starch layer. Cooked rice has good digestion and taste under the usual cooking conditions of polished rice. Actually, dewaxed brown rice is sometimes served in hospitals in Japan.

Brown rice containing the bran layer is thought to be a nutritionally excellent food because of a higher amount of functional components such as vitamin $\mathrm{E}$, vitamin B1, dietary fiber, $\gamma$-oryzanol compared to polished rice (3). Moreover, it is found that the hot water extract of brown rice has a macrophage activating ability (1). This effect is associated with toll like receptor 4 (TLR4) and inhibited by polymyxin $\mathrm{B}$. Thus, the major macrophage activating substance is thought to be lipopolysaccharide (LPS). LPS is a molecule constituting the outer membrane of Gramnegative bacteria. Many edible plants were found to contain significant amount of LPS (4), and it can be said that LPS is a universal functional food component of plants (5).

It has been reported that oral administration of LPS exerts anti-allergic effects $(6,7)$, antitumor effects $(8)$, microvascular promotion (9), and lowering LDL in higher human (10). It is also reported that oral administration of LPS enhances phagocytic activity of peritoneal phagocytes (11), regulatory T cells population in Peyer's patch and mesenteric lymph node (12), and antimicrobial peptide production in intestinal epithelial cells (13). These reports suggest that LPS contained in edible plants is regarded as a functional food component.

The LPS concentration of the bran layer in brown rice was analyzed, and a rich amount of LPS was found to exist in the outer layer of brown rice. LPS amount of water extract of dewaxed brown rice was half of the original brown rice (2). 

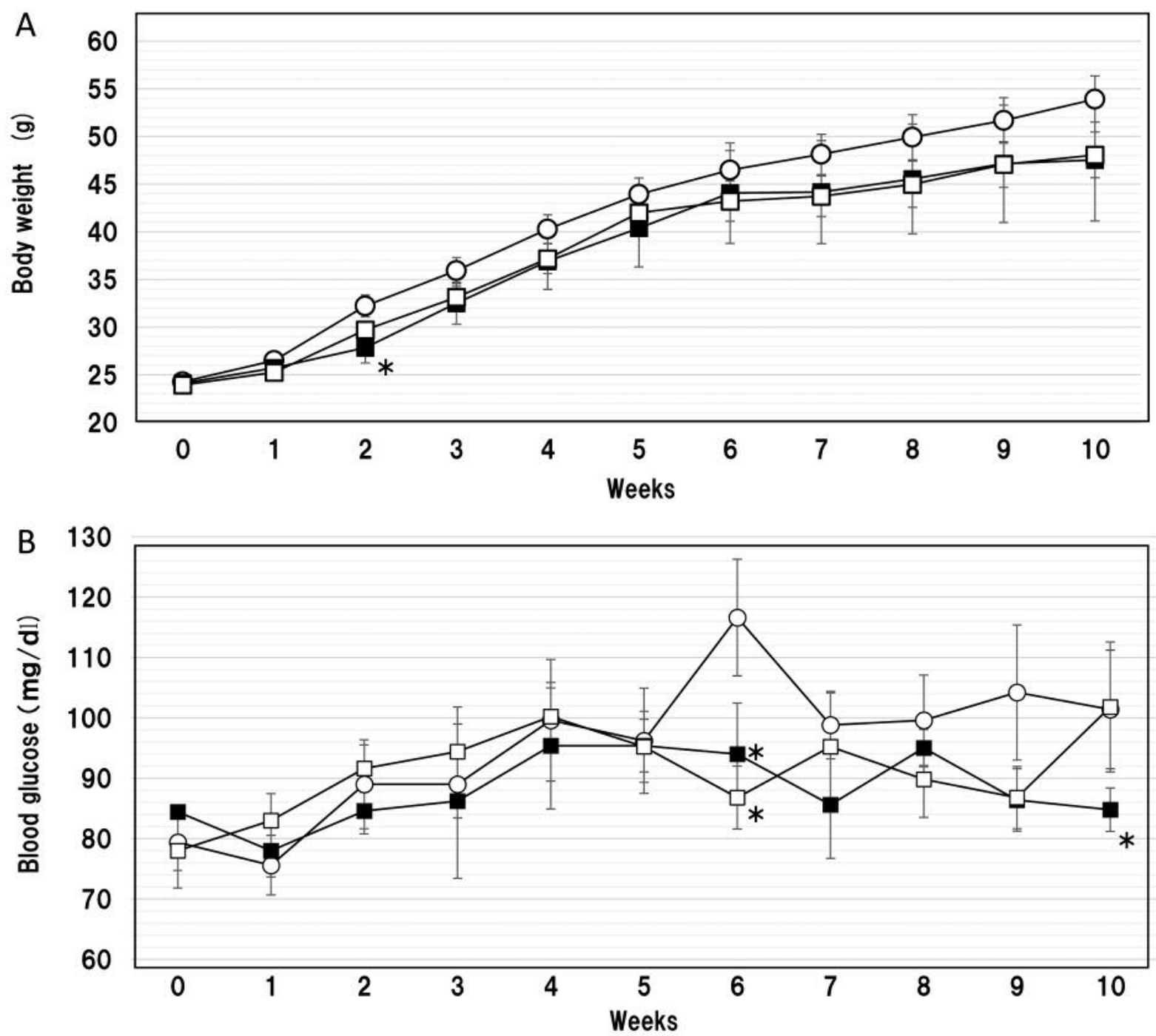

Figure 1. Changes in body weight change and fasting blood glucose level in KK-Ay mice. A: Weight change for 10 weeks after breeding, B: Fasting blood sugar change for 10 weeks after breeding. Open circle: standard diet group (Control), Open square: 50\% polished rice feed group, Closed square: $50 \%$ dewaxed brown rice feed group. Bar means standard deviations. ${ }^{*} p<0.05$ compared to control.

In the present report, as for research of functional analysis of dewaxed brown rice in vivo, obese protection effect was investigated using KK-Ay mice. KK mice are a disease model animal that develops obesity-type non-insulindependent diabetes mellitus (NIDDM), and obesity is moderate even after maturation. $\mathrm{KK}$ is considered a suitable model animal for human NIDDM research.

\section{Materials and Methods}

Sample preparation. Brown rice, dewaxed brown rice and polished rice of Koshihikari (from Nagano prefecture) were prepared using the Saika-style (Muro) rice polishing process in Toyo Rice Corp (Wakayama, Japan). Both types of rice were powdered in a blendermill. For preparation of the diet pellet, American Institute of Nutrition-93 (AIN-93) was used as a standard diet, and $50 \%$ of dewaxed brown rice powder or polished rice powder were mixed with standard diet and pelleted (Reserch Diets, Inc. New Brunswick, NJ, USA). The feed pellet consisted of $8 \%$ ash, $63 \%$ carbohydrate, $17 \%$ protein, $4 \%$ fat and $4.7 \%$ fiber.

Induction of obesity in mice. Following acclimatization, KK/Ay female mice (age 10 weeks, CLEA, Japan, Tokyo) were fed AIN93, 50\% polished rice AIN-93 or 50\% dewaxed brown rice AIN-93 for 10 weeks $(n=5)$. LPS content in AIN-93, 50\% polished rice and dewaxed brown rice were $3.6 \mathrm{ng} / \mathrm{g}, 0.0125 \mu \mathrm{g} / \mathrm{g}$ and $0.246 \mu \mathrm{g} / \mathrm{g}$, respectively.

Food and distilled water were provided ad libitum to mice all through the experiment. Experimental procedures obtained ethical 


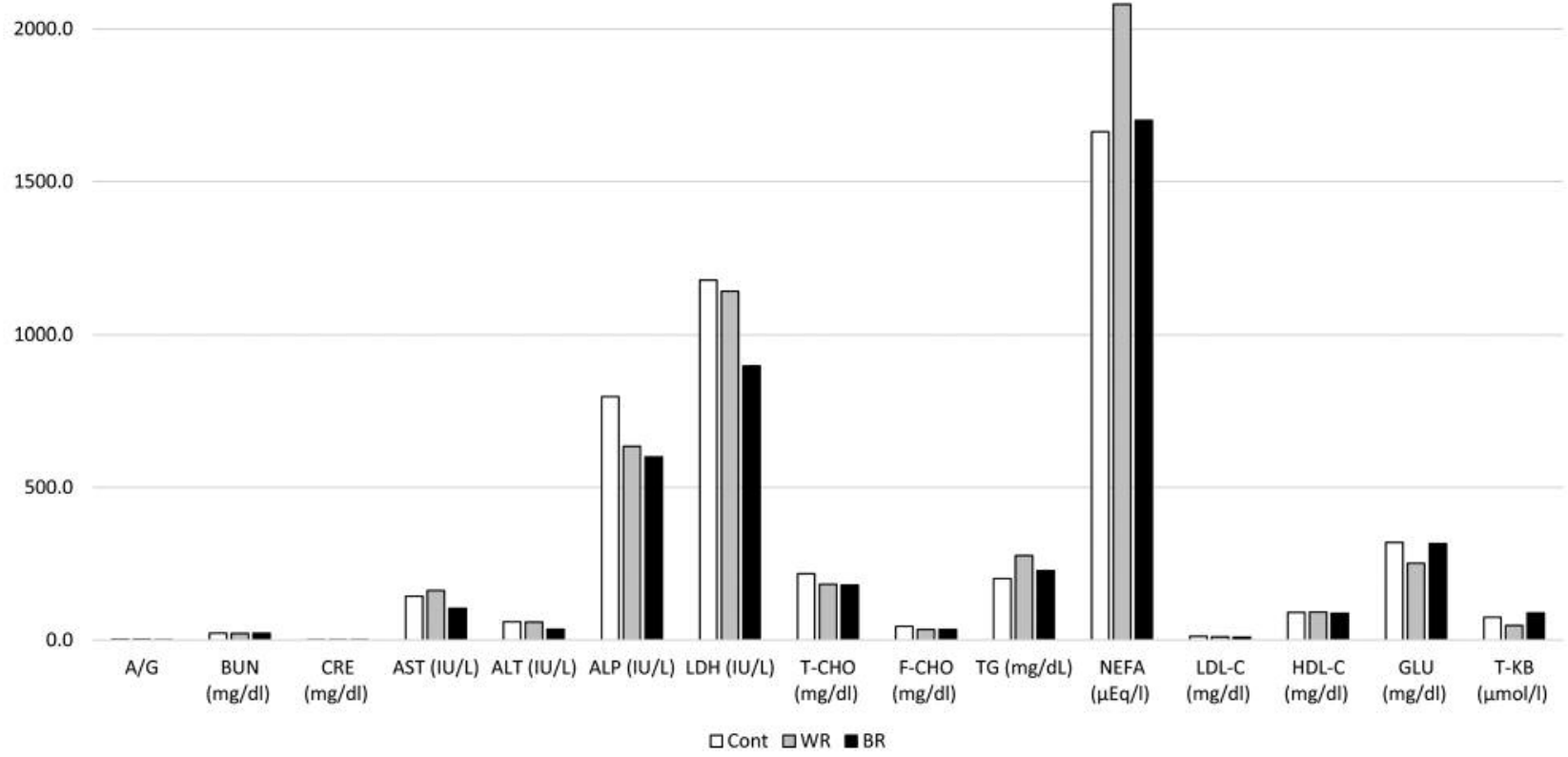

Figure 2. Plasma biochemical marker after ingestion for 10 weeks. A/G: Albumin/globulin ratio; BUN: urea nitrogen; CRE: creatinine; AST: aspartate aminotransferase; ALT: alanine aminotransferase; ALP: alkaline phosphatase; LDH: lactate dehydrogenase; T-CHO: total cholesterol; F-CHO: free cholesterol; TG: triglyceride; NEFA: non-esterified fatty acid; LDL-C: low density lipoprotein cholesterol; HDL-C: high density lipoprotein cholesterol; GLU: glucose; T-KB: total ketone bodies. White column: standard diet group (control), gray column: $50 \%$ polished rice feed group, black column (WR): 50\% dewaxed brown rice feed group (BR).

clearance with reference No. 002 and were conducted in accordance with the guidelines established by the Institutional Animal Care and Use Committee of Control of Innate Immunity, Technology Research Association.

Blood and organ tissue collection. Blood samples were collected after an overnight fast from the tail vein every week. The blood glucose level was analyzed by Accu-chek (Roche DC Japan, Tokyo, Japan). At the end of the treatment, mice were exsanguinated through cardiac puncture, and collected blood was centrifuged for $10 \mathrm{~min}$ at $3500 \mathrm{rpm}$ at room temperature and the supernatants were collected and kept at $-80^{\circ} \mathrm{C}$. The livers were surgically removed, rinsed with saline and their wet weights were measured. The visceral adipose tissue (gonadal fat) was dissected and wet weights were measured.

Biochemical analysis. Plasma biochemical analyses (albumin/globulin ratio, urea nitrogen, creatinine, aspartate aminotransferase, alanine aminotransferase, alkaline phosphatase, lactate dehydrogenase, total cholesterol, free cholesterol, triglyceride, non-esterified fatty acid, low density lipoprotein cholesterol, high density lipoprotein cholesterol, glucose, total ketone bodies) were measured by Oriental Yeast (Tokyo, Japan). The amount of triglyceride and total cholesterol in the liver were measured by Skylight-Biotech (Akita, Japan).
Statistical analysis. Analyses were performed by one-way analysis of variance (ANOVA), followed by Duncan's multiple-range test, using Excel 2008 ver.1.07 (SSRI, Tokyo, Japan). The Dunnett's multiple comparison test was used to compare relative gene expression levels.

\section{Results}

Change in body weight and fasting blood glucose level for 10 weeks ingestion of dewaxed brown rice. KK/Ay mice $(n=5)$ were fed with standard diet (AIN-93), 50\% mixed polished rice powder, $50 \%$ mixed dewaxed brown rice powder for 10 weeks, and the body weight and fasting blood glucose were measured once every week (Figure 1). As compared with the standard diet group, weight loss of about $10 \%$ was observed in the $50 \%$ polished rice group and $50 \%$ dewaxed brown rice group (Figure 1A). In the standard-diet group, fasting blood glucose showed a tendency to increase over the period, and especially high levels of about 100 to $115 \mathrm{mg} / \mathrm{dl}$ were observed in the latter 6-10 weeks (Figure $1 \mathrm{~B})$. On the other hand, fasting blood glucose in the $50 \%$ polished rice group and $50 \%$ dewaxed brown rice group was less than $100 \mathrm{mg} / \mathrm{dl}$ in almost all periods. 
A

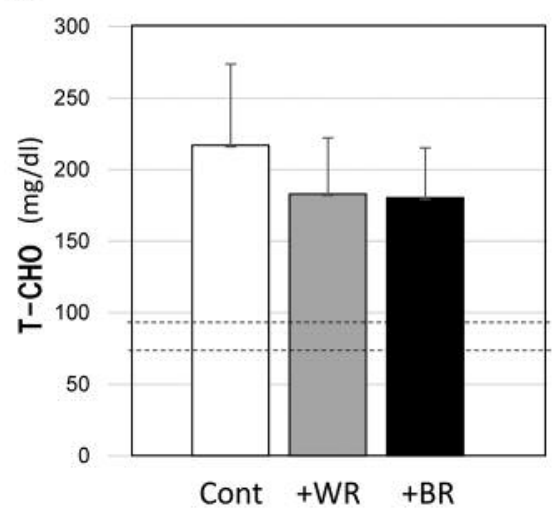

D

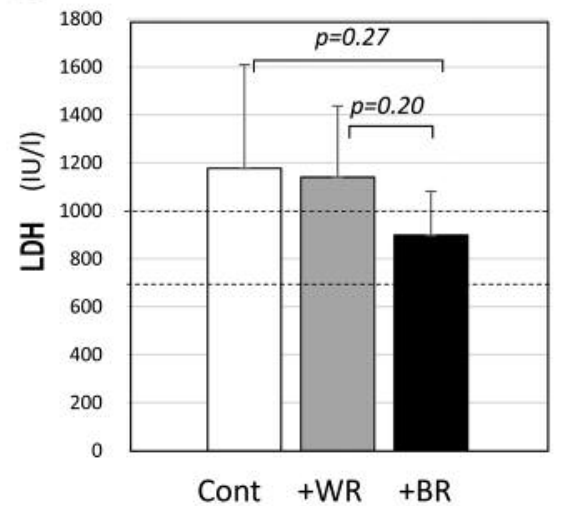

B

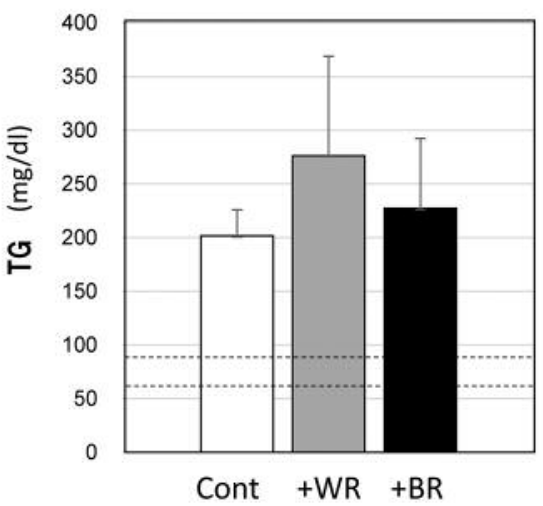

$E$

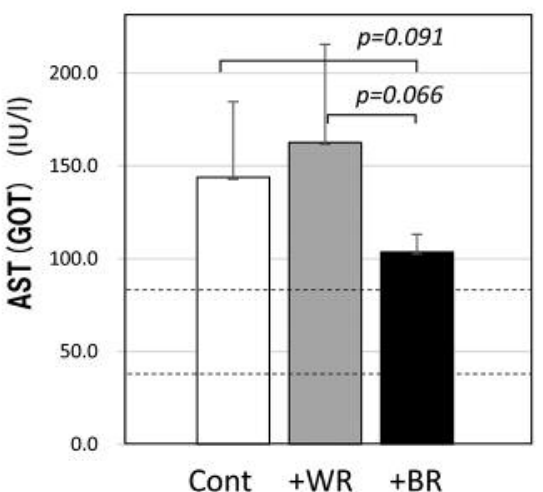

C

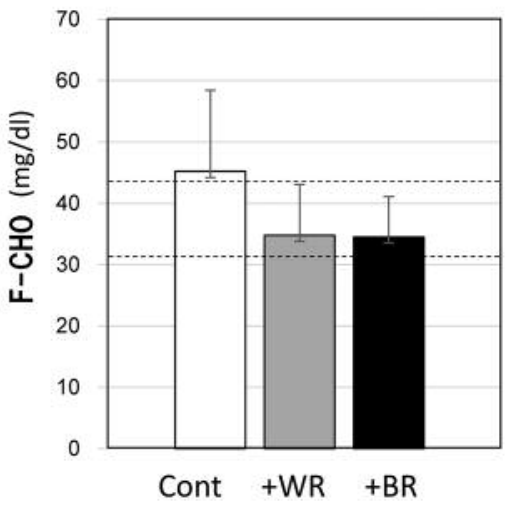

$\mathrm{F}$

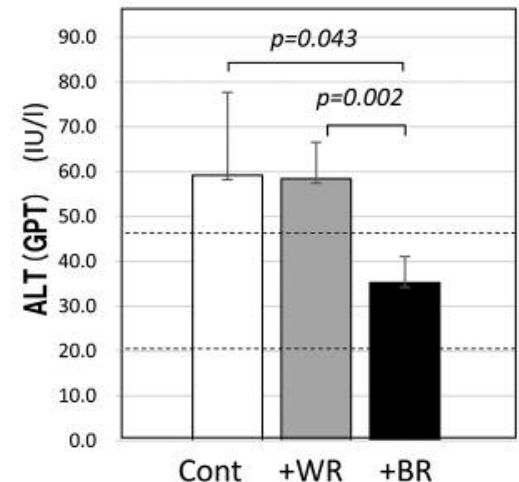

Figure 3. Liver fat marker and condition after 10 weeks ingestion of dewaxed brown rice. A: Plasma T-CHO (total cholesterol), B: Plasma TG (triglyceride), C: Plasma F-CHO(free cholesterol), CD: Plasma LDH (lactate dehydrogenase), E: Plasma AST (GOT) (aspartate aminotransferase), F: Plasma ALT(GPT) (alanine aminotransferase). Columns and bars indicate means and standard deviations, respectively. White column: standarddiet group (control), gray column: 50\% polished rice feed-group, black column (WR): 50\% dewaxed brown rice feed-group (BR).

Biochemical marker after 10 weeks ingestion of dewaxed brown rice. After ten weeks of feeding, KK/Ay mice blood was collected. Biochemical markers of plasma were measured (Figure 2). The lipid markers, T-CHO (Figure 3A), TG (Figure 3B) and F-CHO (Figure 3C) are highly related to obesity. While there was no statistically significant change in these markers, F-CHO showed a tendency to be slightly lower in polished rice and dewaxed brown rice than the standard diet.

The liver markers, LDH (Figure 3D) and AST (Figure 3E) also showed a tendency to be slightly lower in dewaxed brown rice group than polished rice and standard diet group. ALT (Figure 3F) was significantly decreased to almost normal range in the dewaxed brown rice group.

Liver fat condition after 10 weeks ingestion of dewaxed brown rice. After feeding for ten weeks, KK/Ay mice gonadal fat and liver were collected and weighed. The weights of gonadal fat and liver are shown in Figure 4A and $4 \mathrm{~B}$. There was no difference in gonadal fat weight among standard diet, $50 \%$ polished rice and $50 \%$ dewaxed brown rice group. In terms of liver weight, it reached approximately $7.8 \%$ of the body weight in the standard diet group. This liver/body weight ratio indicates fatty liver because this ratio was reaching 2.1 times higher compared to that of normal female C57BL/6 mice (liver/body weight ratio is $3.8 \%, 10$ weeks, Japan SLC data). On the other hand, the $50 \%$ polished rice and $50 \%$ dewaxed brown rice diet groups were about $5.5 \%$ of the body weight which was about $28 \%$ lower than that of the standard diet group (not significant).

Lipids were extracted from the liver, and TG (Figure 4C) and T-CHO (Figure 4D) were measured. Compared to the standard diet, TG content in the liver of $50 \%$ polished rice group was decreased $(67 \%)$, but a more significant decrease was observed in the $50 \%$ dewaxed brown rice diet group 

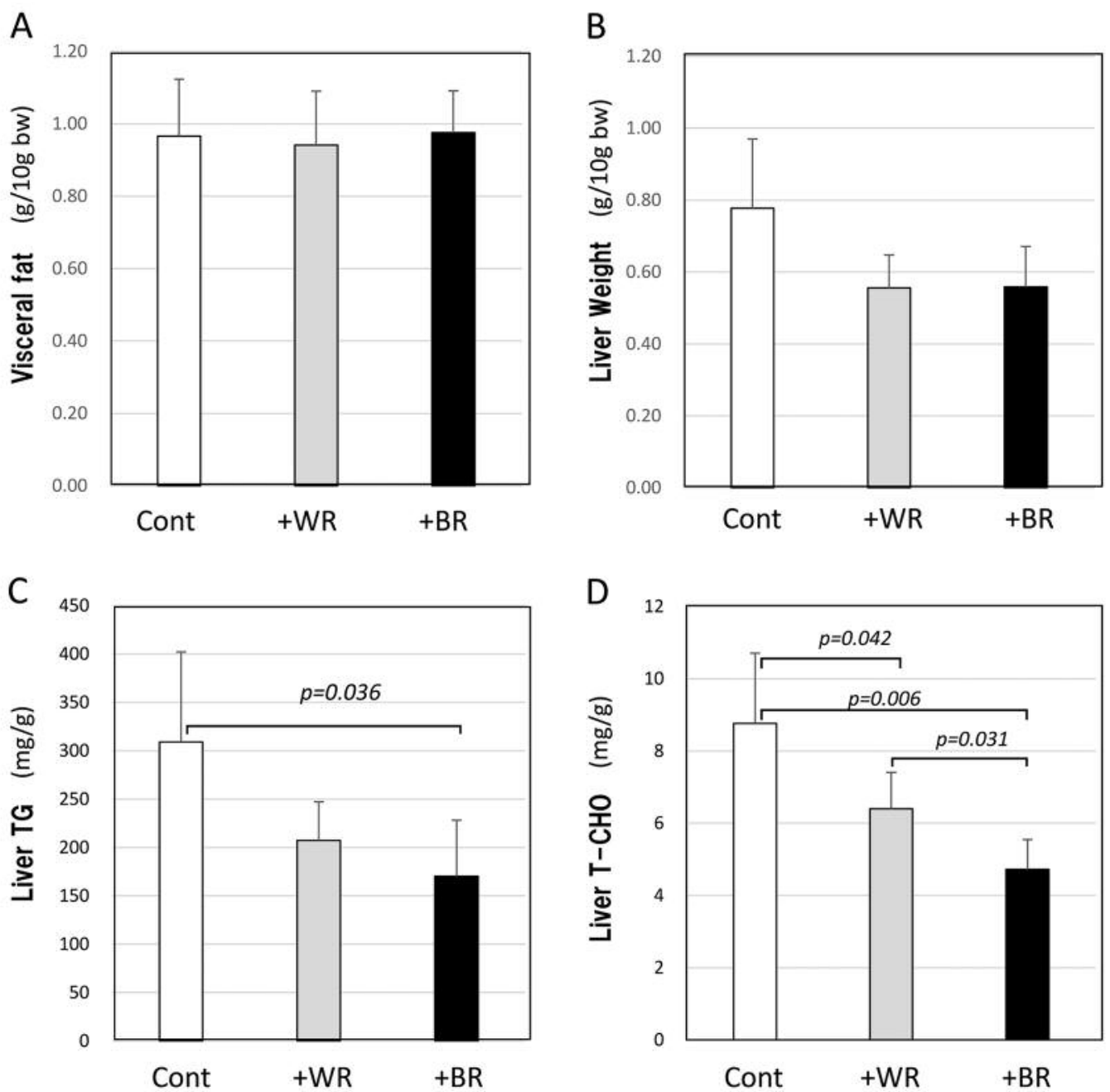

Figure 4. Liver fat content after 10 weeks ingestion of dewaxed brown rice. A: Visceral fat (Gonadal fat), B: liver weight, C: Triglyceride content in liver, D: total cholesterol content in the liver. Columns and bars indicate means and standard deviations, respectively. White column: standarddiet group (control), gray column: 50\% polished rice feed-group, black column (WR): $50 \%$ dewaxed brown rice feed-group (BR).

(58\%). T-CHO in the liver was significantly reduced with the $50 \%$ polished rice $(73 \%)$ compared to the standard diet group. In addition, $\mathrm{T}-\mathrm{CHO}$ in the $50 \%$ dewaxed brown rice group was significantly decreased compared with the standard group (54\%) and 50\% polished rice diet group.

\section{Discussion}

A new rice milling technique which could remove the wax layer on brown rice was established by Toyo Rice (Wakayama, Japan). This apparatus can produce dewaxed brown rice which has high water absorbency compared to that of original brown rice. Therefore, compared to conventional brown rice, the immersion time is less, cooking is as easy as ordinary polished rice. Moreover, it is characterized by good taste for many people. In this study, the anti-obesity effect was evaluated, in particular the preventive effect of fatty liver, among the health maintenance function of dewaxed brown rice, and the significant suppressive effect of TG and T-CHO in the liver were demonstrated.

In recent years, the treatment of non-alcoholic fatty liver disease is considered indispensable for the prevention and management of Type 2 diabetes mellitus (14). Fatty liver is a state in which triglyceride (TG) and cholesterol accumulate in the liver, and fat vacuoles are formed in excess. Progression of fatty liver causes liver cirrhosis and liver cancer. It is possible that ingestion of dewaxed brown rice reduces hepatic fat mass of the obese mouse model and may prevent lifestyle diseases induced by obesity. 
Several reports on germinated brown rice have been generated for their immune-stimulating effect (15). Germinated brown rice (GBR) is considered a whole food because only the outermost layer i.e. the hull of the rice kernel is removed which causes the least damage to its nutritional value. Brown rice can be soaked in water at $30^{\circ} \mathrm{C}$ for germination. Soaking for $3 \mathrm{~h}$ and sprouting for $21 \mathrm{~h}$ has been found to be optimal for the highest gammaaminobutyric acid (GABA) content in GBR, which is the main reason behind the popularity of GBR. However, the cost of commercially available GBR is extremely high. Thus, it is not widespread in Japan. On the other hand, the dewaxed brown rice has similar water absorbency and cost to polished rice, therefore there is the ability to improve the problem of brown rice. The amount of functional ingredients in the dewaxed brown rice used in this study is not greatly reduced compared to brown rice. For example, LPS amount is about half of the original brown rice (2).

It is well known that various functional constituents are present in the bran layer of rice such as vitamins, minerals, dietary fiber, $\gamma$-oryzanol etc. For example, $\gamma$-oryzanol is used in Japan as a drug for treating dyslipidemia (by suppression of LDL cholesterol) and psychosomatic disorders (by suppression of central nervous system with catecholamine metabolism in the hypothalamus) $(16,17)$. Dietary fiber promotes bowel movements due to an increase and improvement in intestinal microbiota (18). However, it is still unknown what components contained in brown rice contribute to this phenomenon. According to Felix et al., 4 weeks administration of $80 \%$ ethanol extract of brown rice or black rice to KK-Ay mice showed significant suppression of TG amount in the liver compared to the control group (19). On the other hand, they demonstrated that the amount of TG in blood was increased predominantly. In this study, TG in the blood was not altered by ingestion of the dewaxed brown rice, but the TG in the liver decreased. These results suggest that the lipid components may contribute in part for the decrease in liver TG in the dewaxed brown rice fed mice. They also demonstrated that this extract prevents liver damage by suppression of oxidative stress due to the action of PPAR $\alpha$ in KK-Ay mice (19). Since $\gamma$-oryzanol and LPS in the rice bran have anti-inflammatory actions $(20,21)$, it is believed that it may contribute to avoidance of oxidative stress in the liver, but further details will be analyzed in the future.

\section{Conflicts of Interest}

This study was partly funded by SIP. TS and NN are employees of Toyo Rice Corp. TN and CK are employees of Macrophi Inc. The sponsor had no control over the interpretation, writing, or publication of this work. The corresponding Author had full access to all the data in the study and had final responsibility for the decision to submit for publication.

\section{Acknowledgements}

This work was partly supported by the Council for Science, Technology and Innovation (CSTI), Cross-ministerial Strategic Innovation Promotion Program (SIP), "Technologies for creating next-generation agriculture, forestry and fisheries" (funding agency: Bio-oriented Technology Research Advancement Institution, NARO).

\section{References}

1 Ho JN, Son ME, Lim WC, Lim ST and Cho HY: Anti-obesity effects of germinated brown rice extract through downregulation of lipogenic genes in high fat diet-induced obese mice. Biosci Biotechnol Biochem 76: 1068-1074, 2012.

2 Inagawa $H$, Saika $T$, Nisizawa $T$, Kohchi $C$, Uenobe $M$ and Soma G: Dewaxed Brown Rice Contains a Significant Amount of Lipopolysaccharide Pointing to Macrophage Activation via TLRs. Anticancer Res 36: 3599-3605, 2016.

3 Sharif MK, Butt MS, Anjum FM and Khan SH: Rice bran: a novel functional ingredient. Crit Rev Food Sci Nutr 54: 807-816, 2014.

4 Inagawa $\mathrm{H}$, Nishizawa T, Tsukioka D, Suda T, Chiba Y, Okutomi T, Morikawa A, Soma G and Mizuno D: Homeostasis as regulated by activated macrophage. II. LPS of plant origin other than wheat flour and their concomitant bacteria. Chem Pharm Bull (Tokyo) 40: 994-997, 1992.

5 Soma G, Inagawa H, Nishizawa T, Yoshioka N, Taniguchi $Y$ and Kohchi C: Preventative and therapeutic potential of lipopolysaccharide derived from edible gram-negative bacteria to various diseases. Current Drug Therapy 3: 22-32, 2008.

6 Wakame $\mathrm{K}$, Komatsu $\mathrm{K}$, Inagawa $\mathrm{H}$ and Nishizawa $\mathrm{T}$ : Immunopotentiator from pantoea agglomerans prevents atopic dermatitis induced by dermatophagoides farinae extract in NC/Nga Mouse. Anticancer Res 35: 4501-4508, 2015.

7 Amano S, Inagawa H, Nakata Y, Ohmori M, Kohchi C and Soma G: Oral administration of lipopolysaccharide of acetic acid bacteria protects pollen allergy in a murine model. Anticancer Res 35: 4509-4514, 2015.

8 Morishima $\mathrm{A}$ and Inagawa $\mathrm{H}$ : Clinical effects of orally administered lipopolysaccharide derived from Pantoea agglomerans on malignant tumors. Anticancer Res 36: 3747-3751, 2016.

9 Nakata Y, Kohchi C, Ogawa K, Nakamoto T, Yoshimura H and Soma GI: Effects of 3 months continuous intake of supplement containing Pantoea agglomerans LPS to maintain normal bloodstream in adults: Parallel double-blind randomized controlled study. Food Sci Nutr 20: 197-206, 2017.

10 Nakata K, Nakata Y, Inagawa H, Nakamoto T, Yoshimura H and Soma G: Pantoea agglomerans lipopolysaccharide maintains bone density in premenopausal women: a randomized, double-blind, placebo-controlled trial. Food Sci Nutr 2: 638-646, 2014.

11 Inagawa H, Kobayashi Y, Kohchi C, Zhang R, Shibasaki Y and Soma G: Primed activation of macrophages by oral administration of lipopolysaccharide derived from Pantoea agglomerans. In Vivo 30: 205-211, 2016.

12 Hrncir T, Stepankova R, Kozakova H, Hudcovic $T$ and Tlaskalova-Hogenova $\mathrm{H}$ : Gut microbiota and lipopolysaccharide content of the diet influence development of regulatory $\mathrm{T}$ cells: studies in germ-free mice. BMC Immunol 9: 65, 2008. 
13 Brandl K1, Plitas G, Mihu CN, Ubeda C, Jia T, Fleisher M, Schnabl B, DeMatteo RP and Pamer EG: Vancomycin-resistant enterococci exploit antibiotic-induced innate immune deficits. Nature 455: 804-807, 2008.

14 Portillo-Sanchez P and Cus K: Treatment of Nonalcoholic Fatty Liver Disease (NAFLD) in patients with Type 2 Diabetes Mellitus Clin Diabetes Endocrinol 2: 9, 2016.

15 Patil SB and Khan MK: Germinated brown rice as a value added rice product: A review. J Food Sci Technol 48: 661-617, 2011.

16 Ha SJ, Park J, Lee J, Song KM, Um MY, Cho S and Jung SK: Rice bran supplement prevents UVB-induced skin photoaging in vivo. Biosci Biotechnol Biochem 82: 320-328, 2018.

17 Goufo P and Trindade H: Rice antioxidants: phenolic acids, flavonoids, anthocyanins, proanthocyanidins, tocopherols, tocotrienols, $\gamma$-oryzanol, and phytic acid. Food Sci Nutr 2: 75104, 2014.

18 Han M, Wang C, Liu P, Li D, Li Y and Ma X: Dietary Fiber Gap and Host Gut Microbiota. Protein Pept Lett 24: 388-396, 2017.
19 Felix AD, Takahashi N, Takahashi M, Katsumata-Tsuboi R, Satoh R, Soon Hui T, Miyajima K, Nakae D, Inoue H and Uehara M: Extracts of black and brown rice powders improve hepatic lipid accumulation via the activation of PPAR $\alpha$ in obese and diabetic model mice. Biosci Biotechnol Biochem 81: 2209-2211, 2017.

20 Rao YP, Sugasini D and Lokesh BR: Dietary gamma oryzanol plays a significant role in the anti-inflammatory activity of rice bran oil by decreasing pro-inflammatory mediators secreted by peritoneal macrophages of rats. Biochem Biophys Res Commun 479: 747-752, 2016.

21 Caramalho I, Lopes-Carvalho T, Ostler D, Zelenay S and Haury $\mathrm{M}$ and Demengeot J: Regulatory T cells selectively express tolllike receptors and are activated by lipopolysaccharide. J Exp Med 197: 403-411, 2003.

Received April 11, 2018

Revised May 31, 2018

Accepted June 4, 2018 